MATEC Web of Conferences 13, 05012 (2014)

DOI: $10.1051 /$ matecconf/ 20141305012

(C) Owned by the authors, published by EDP Sciences, 2014

\title{
A Review of $\mathrm{CO}_{2}$ Corrosion Inhibition by Imidazoline-based Inhibitor
}

\author{
Rafida Ahmad Jaal ${ }^{1, a}$, Mokhtar Che Ismail ${ }^{1}$ and Bambang Ariwahjoedi ${ }^{2}$ \\ ${ }^{1}$ Centre for Corrosion Research, Universiti Teknologi PETRONAS, Bandar Seri Iskandar, 31750 \\ Tronoh, Perak, Malaysia \\ ${ }^{2}$ Universiti Teknologi PETRONAS, Bandar Seri Iskandar, 31750 Tronoh, Perak, Malaysia
}

\begin{abstract}
Carbon dioxide $\left(\mathrm{CO}_{2}\right)$ corrosion is one of the most significant forms of attack in the oil and gas production and transportation systems. Corrosion inhibitors have been widely used in an effort to reduce the detrimental effect of the corrosion process. Different types of corrosion inhibitors have been applied for this purpose. The most frequent is the imidazoline-based inhibitors (IM), owing to their good adsorption characteristics and film-forming capability. Albeit their extensive use, their inhibition mechanism is not fully understood. This paper highlights the inhibition mechanism of IM and also the factors that contribute to its inhibition mechanism.
\end{abstract}

\section{Introduction}

Corrosion is defined as the destruction or deterioration of a material or its properties because of reaction with its environment $[1,2]$. Carbon dioxide $\left(\mathrm{CO}_{2}\right)$ corrosion is one of the most prevalent forms of attack encountered in oil and gas industry, significantly in the production and transportation systems. The main concern is the failure of equipment caused by $\mathrm{CO}_{2}$ corrosion, particularly the main downhole tubing and transmission pipelines, which results in disruption of the oil and gas production. Failures related to corrosion comprise over $25 \%$ of failures experienced in this industry, where $28 \%$ of it is $\mathrm{CO}_{2}$ related [3]. The costs associated with corrosion cannot be eliminated entirely and it is almost impossible to completely prevent corrosion. Therefore the most economical solution is to control the rate of the corrosion. Corrosion inhibitors have been considered as the first line defense against internal corrosion of pipelines [4]. In conjunction with the use of carbon steel, corrosion inhibitors have been recognized as the most practical and cost-effective option. The most common corrosion inhibitor used in oil and gas industry is imidazoline-based formulation.

\section{$2 \mathrm{CO}_{2}$ Corrosion Inhibitors}

There are two types of $\mathrm{CO}_{2}$ corrosion inhibitors used in oil and gas industry. The film-type corrosion inhibitors are mainly inorganic, such as chromate and nitrate. The disadvantages of inorganic inhibitors are that they are required in large dosages, apart from their poor feasibility, high treatment frequency and unsatisfactory effectiveness $[4,5]$. The adsorption-type inhibitors reduce corrosion rate

\footnotetext{
${ }^{\text {a }}$ Corresponding author : fida.jaal@gmail.com
}

This is an Open Access article distributed under the terms of the Creative Commons Attribution License 2.0, which permits unrestricted use, distribution, and reproduction in any medium, provided the original work is properly cited. 
by forming layers of hydrophobic film on metal surface, which hinders transfer of substance and charges related to corrosion reactions. They are usually organic and the most widely used are the nitrogen-containing compounds, for example amines, amides, quaternary ammonium salts, imidazolines and their derivatives, salts of nitrogenous molecules with carboxylic acids, polyoxyalkylated nitogen-containing compounds, nitorgen heterocyclics and compounds containing phosphorus, sulfur and oxygen [6-8]. These inhibitors are categorized as surfactants due to their amphipathic, lipid-like molecular structure. They preferentially adsorb onto surfaces or interface in a system and modify the surface and interfacial free energies [6].

\section{Imidazoline-based Inhibitor}

The imidazoline-based inhibitors (IM) are most frequently used to protect pipelines from $\mathrm{CO}_{2}$ corrosion because of its good adsorption characteristics and its ability to form film on metal surface [9]. The molecular structure of IM can be divided into three substructures, as seen in Figure $1[5,6]$.

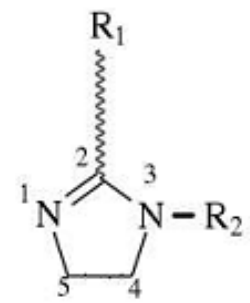

Figure 1. Scheme of an imidazoline molecule

The hydrophilic, polar head group is a nitrogen-bearing five-membered ring. The long hydrocarbon chain (R1) forms hydrophobic layers in solution that hinders the contact between the corrosive medium and the metal surface. Thus it is also known as the hydrophobic branched chain. The pendant side chain with an active functional group (R2) is also referred to as the hydrophilic branched chain. It mostly contains nitrogen, oxygen and other heteroatoms, which can result in stable chemical adsorption on the surface. The IM derivatives behave as cationic surfactants, which are determined by the nature of hydrocarbon or substituent groups attached to carbon or nitrogen atom of the IM ring [7].

\subsection{Inhibition Mechanism of Imidazoline}

The inhibiting mechanism of IM is attributed to their adsorption to the metal-solution interface. There are two types of adsorption that can be distinguished from their mechanism. The physical adsorption involves electrostatic attractive forces between ionic charges on the inhibitor molecules and the charged metal surface. The second type of adsorption is the chemisorption, which is a result from sharing or transferring of charge from inhibitor molecules to metal surface in order to form a chemical bond [7]. IM molecules have unshared electron pairs on nitrogen atoms, which can interact with the dorbitals of iron. This interaction forms a coordinate-type bond [10].

Irrespective of the adsorption mechanism, the attractions or chemical bonds are between the hydrophilic head and steel surface. The adsorption process can be viewed as a quasi-substitution process between the inhibitor molecules in the solution and the adsorbed water molecules on metal surface [11]. The IM molecules have larger adsorption energy than water molecules. Therefore IM molecules adsorb preferentially on steel surface in an aqueous media [12].

The molecular structure of IMs has a significant role in their performance as corrosion inhibitor [13]. The pronounced effect of the hydrocarbon chain R1 is the formation of protective film at the 
metal-solution interface. Jovancicevic et. al [14] stated that the length of R1 in the range of C12 to C20 has significant importance in the corrosion inhibition of IMs. In addition, no evident inhibition was observed with the application of C8 imidazoline. López et. al [8] have stated in their work that presence of long R1 could be related to formation of a more protective, yet porous barrier against aggressive ions from the solution due to the interactions between the chains. On the other hand, Ramachandran et. al. [15] reported that inhibitors with chain length of $\mathrm{C}<12$ exhibit poor performance. A number of corrosion inhibitors can be used at low concentration without efficiency lost due to their large molecular structures. However, the efficiency may reduce if the inhibitor concentration is increased. This is attributed to the tendency of the inhibitor molecules to repel each other [11]. The pendant side chain R2 likewise can significantly influence the molecular reactivity and binding strength between the inhibitor self-assembled monolayer and iron surface, and also the compactness of the self-assembled layer [16].

\subsection{Factors Affecting Inhibition Performance}

Factors that affect the adsorption process, efficiency and inhibition mechanism can be divided into a few categories [17-19].

The first factor is related to the inhibitor itself, such as its electronic and structural characteristics, composition, concentration, film forming characteristic, interaction with steel surface, etc. Inhibitors with large molecular size and high electron density on the adsorption centres may possess high inhibition efficiency [18]. A surfactant with long hydrocarbon chain will adsorb to each other to form a monolayer at the metal-solution interface [10]. Xiong et. al [20] studied the properties of adsorbed inhibitor using in-situ atomic force microscopy (AFM). The results showed that a monolayer was formed when the applied inhibitor concentration was below the critical micelle concentration (CMC), and a bilayer at concentration above CMC. CMC is the concentration, at which the surfactant molecules spontaneously agglomerate together to form small colloid particles called micelles within the bulk liquid. Formation of the first adsorbed layer was due to electrostatic interactions of positive ions on negatively charged surfaces. In bilayer arrangement, the hydrocarbon chains are facing each other, while the hydrophilic groups point towards the solution and the steel surface.

The second category is related to nature and condition of the steel surface, its microstructure and also presence of surface film. According to Ramachandran et. al [21], the blank and inhibited corrosion rates are strongly influenced by the corrosion product layer formation and the incorporation of the inhibitor through the corrosion product layer. Zhang et. al. [12] observed that the oriental angles of IM rings on iron carbonate surface were smaller than that on Fe surface. This indicates that the interaction of IM ring with iron carbonate was stronger than with Fe. In a study conducted by Gulbrandsen et. al [22], the effect of precorrosion is also influenced by steel properties. For X65 specimen, the inhibitor performed efficiently even after 14-day precorrosion period. Yet the inhibitor acted slower with increasing precorrosion time. For St52 specimen, the inhibitor was not protecting the steel at all, even though the latter was precorroded at the same duration as the former. Cathodic inhibitors perform well on steel microstructures consisting of ferrite and pearlite phases, while anodic inhibitors on ferritic or martensitic structures [23].

Other factors are related to the conditions under which the inhibitor is applied, for example, temperature, pressure, $\mathrm{pH}$, flow velocity, composition of the aggressive environment, etc. $\mathrm{CO}_{2}$ saturated solutions have a $\mathrm{pH}$ value in the range of 4.7 to 5.2. The neutralization of amine group with the weak carbonic acid is reversible, which means that IM might adsorb as a neutral molecule or as a carbonate salt [10]. The adsorption and desorption processes of inhibitor molecules equilibrate at a particular temperature. Increasing the temperature causes the desorption rate to be higher than that of adsorption, resulting in lower efficiency [13]. Inhibitor film can be removed from metal surface at some critical fluid velocity, which is dependent on inhibitor concentration and type. However the study revealed that the stress required to physically remove an inhibitor film is at least three orders of magnitude higher than the typical wall shear stress seen in turbulent pipe flow [20]. This shows that the mechanical forces of the fluid flow alone are not enough to remove the film. Chloride ions in the 
solution may assist the inhibitor molecule in the adsorption process. Steel surface is positively charged in acid solution. As chloride ions have small degree of hydration, they could bring excess negative charges in the vicinity of the metal-solution interface, promoting more adsorption of the positively charged inhibitor molecules through electrostatic interactions [4]. On the other hand, Nesic et. al [17] stated that it is possible that the chloride ions, which are a weak inhibitor, competed with the inhibitor molecules for adsorption onto metal surface.

\section{Summary}

Corrosion inhibitors have been one of the most preferred means of controlling $\mathrm{CO}_{2}$ corrosion. Different types of inhibitors with different compositions have been formulated in order to achieve the best inhibition performance. In selecting inhibitors, various factors must be taken into consideration as they can affect the inhibition mechanism in many ways. This has become more apparent because in the quest for new oil and gas sources, operational activities have moved to harsher environments. Therefore, understanding the functioning of the corrosion inhibitor will benefit in the development of better products for a broad range of conditions.

\section{References}

1. G. H. Koch, M. P. H. Brongers, N. G. Thompson, Y. P. Virmani, J. H. Payer, Corrosion Costs and Preventive Strategies In the United States; NACE International.

2. M. G. Fontana, Corrosion Engineering, Third edit., McGraw-Hill Book Company (1987)

3. M. B. Kermani, D. Harrop, SPE Prod. Facil., 11, 186-190 (1996)

4. M. A. Ibraheem, A. E. A. El Sayed Fouda, M. T. Rashad, F. Nagy Sabbahy, ISRN Metall. 2012, 2012, 1-15 (2012)

5. Y. P. Cheng, Z. L. Li, H. S. Bi, Y. X. Song, Adv. Mater. Res., 734-737, 1240-1245(2013)

6. H. M. A. El-lateef, V. M. Abbasov, L. I. Aliyeva, T. A. Ismayilov, Chem. J., 02, 52-63 (2012)

7. M. Heydari, M. Javidi, Corros. Sci., 61, 148-155 (2012)

8. D. A. López, S. N. Simison, S. R. de Sánchez, Corros. Sci., 47, 735-755(2005)

9. X. Zhang, F. Wang, Y. He, Y. Du, Corros. Sci., 43, 1417-1431(2001)

10. M. W. S. Jawich, G. A. Oweimreen, S. A. Ali, Corros. Sci., 65, 104-112 (2012)

11. F. Farelas, A. Ramirez, Int. J. Electrochem. Sci., 5, 797-814 (2010)

12. J. Zhang, J. Liu, W. Yu, Y. Yan, L. You, L. Liu, Corros. Sci., 52, 2059-2065(2010)

13. A. J. A. Nasser, M. A. Sathiq, Int. J. Eng. Sci. Technol., 2, 6417-6426 (2010)

14. V. Jovancicevic, S. Ramachandran, P. Prince, CORROSION 98, 1-17 (1998)

15. S. Ramachandran, B. Tsai, M. Blanco, H. Chen, Y. Tang, W. A. Goddard, Langmuir, 12, 64196428 (1996)

16. J. Zhang, G. Qiao, S. Hu, Y. Yan, Z. Ren, L. Yu, Corros. Sci., 53, 147-152 (2011)

17. S. Nesic, W. Wilhelmsen, S. Skjerve, S. M. Hesjevik, Proceedings of the 8th European Synposium on Corrosion Inhibitors, 1163-1192 (1995)

18. V. M. Abbasov, A. R. Ezizbeyli, R. A. Jafarova, S. Y. Hajiyeva, E. E. Gasimov, Chem. J., 02, 194-198 (2012)

19. G. Karthik, M. Sundaravadivelu, ISRN Electrochem., 2013, 1-10 (2013)

20. Y. Xiong, B. Brown, B. Kinsella, S. Nesic, A. Pailleret, CORROSION 2013, 1-22 (2013)

21. S. Ramachandran, Y. S. Ahn, M. Greaves, V. Jovancicevic, J. Bassett, CORROSION 2006, 1-15 (2006)

22. E. Gulbrandsen, A. Stangeland, T. Burchardt, S. Nesic, S. M. Hesjevik, S. Skjfrve, B. Sundfer, CORROSION 98, 1-20 (1998)

23. A. Khavasfar, M. H. Moayed, A. H. Jafari, IJE Trans. A: Basics, 20, 35-44 (2007) 